06;08

\title{
Кинетика роста планарных нитевидных нанокристаллов
}

\author{
(С) В.Г. Дубровский' , И.В. Штром² \\ ${ }^{1}$ Университет ИТМО, Санкт-Петербург, Россия \\ ${ }^{2}$ Санкт-Петербургский государственный университет, Санкт-Петербург, Россия \\ E-mail: dubrovskii@mail.ioffe.ru
}

Поступило в Редакцию 30 июня 2020 г.

В окончательной редакции 14 июля 2020 г.

Принято к публикации 15 июля 2020 г.

Получено приближенное аналитическое уравнение, описывающее закон удлинения полупроводниковых нитевидных нанокристаллов, растущих по механизму пар-жидкость-кристалл в плоскости подложки. Проведен теоретический анализ различных режимов роста в зависимости от радиуса нитевидного нанокристалла $R$ и условий эпитаксиального осаждения. Показано, что скорость роста планарных нитевидных нанокристаллов может лимитироваться либо эффектом Гиббса-Томсона (при малых размерах капли катализатора), либо диффузией адатомов с поверхности подложки (при увеличении радиуса кристалла). Зависимость диффузионно-лимитированной скорости роста от $R$ имеет вид $R^{-m}$, где степенной показатель принимает значения $1,3 / 2$ или 2 в зависимости от характера поверхностной диффузии.

Ключевые слова: планарный нитевидный нанокристалл, механизм роста пар-жидкость-кристалл, поверхностная диффузия, эффект Гиббса-Томсона.

DOI: 10.21883/PJTF.2020.20.50149.18440

Полупроводниковые нитевидные нанокристаллы (ННК) являются перспективными „строительными блоками“ для фундаментальных исследований и приложений в области наноэлектроники и нанофотоники [1]. Вертикальные ННК, растущие в направлении $\langle 111\rangle$ перпендикулярно поверхности подложки, синтезируются различными эпитаксиальными методами по механизму пар-жидкость-кристалл (ПЖК) [2], где в качестве катализаторов используются Au [2] или металлы III группы (при автокаталитическом ПЖК-росте НHК GaAs [3]). Кинетическая теория роста полупроводниковых ННК, в особенности III-V ННК, довольно хорошо разработана (см., например, обзор [4]). Практические приложения наногетероструктур на основе ННК, в том числе совмещенных с кремниевой электронной платформой, в ряде случаев осложняются их вертикальной геометрией, что вызывает сложности при изготовлении верхнего контакта и другие проблемы. Поэтому большой интерес представляют планарные ННК, выращиваемые на поверхности подложки [5-12]. Системы полупроводниковых материалов, в которых были синтезированы планарные ННК на различных поверхностях, включают $\mathrm{Si}[5]$, соединения III-V [6,7], III-N [8], II-VI [9-11] и оксиды [12]. В работах $[11,13,14]$ была предпринята попытка обобщения известных из теории роста вертикальных ННК моделей на случай планарного роста. Целью настоящей работы является вывод и исследование кинетического уравнения для скорости роста планарных ННК с учетом эффекта Гиббса-Томсона в капле [15] и различных механизмов диффузии адатомов [16,17].

Рассмотрим процесс роста планарного ННК на плоской поверхности подложки, представленный на рис. 1. Пусть ННК представляет собой полуцилиндр радиу- ca $R$, лежащий на поверхности, а капля с одного конца ННК, направляющая его горизонтальный рост, четверть сферы того же радиуса. Эффективный поток элемента, лимитирующего рост есть $I\left(\right.$ в $\left.\mathrm{nm}^{-2} \cdot \mathrm{s}^{-1}\right)$; в случае III-V ННК таким элементом является металл III группы, адатомы которого способны мигрировать по поверхности. Для определенности в дальнейшем рассматриваем случай роста ННК из газовых прекурсоров, тогда поток $I$ включает эффективность соответствующей химической реакции. В предположении, что скорость роста ННК определяется материальным транспортом, а не нуклеацией на границе жидкость-кристалл $[18,19]$, $d L / d t$ содержит четыре вклада: 1) адсорбция-десорбция из газовой фазы непосредственно на поверхности капли; 2) диффузия атомов с поверхности подложки на поверхность капли; 3 ) диффузия атомов с поверхности подложки сначала к поверхности ННК, а затем вдоль последней в каплю; 4) адсорбция на боковой поверхности ННК с последующей диффузией в каплю. В общем случае имеем

$$
\frac{d L}{d t}=2 \Omega\left(I-\frac{n_{l}}{\tau_{l}}+\frac{j_{d i f f}^{s}}{\pi R^{2}}+\frac{j_{d i f f}^{f}}{\pi R^{2}}\right),
$$

где $\Omega$ - элементарный объем в кристалле, $n_{l}-$ эффективная концентрация атомов в поверхностном слое капли, измеряемая в $\mathrm{nm}^{-2}, \tau_{l}-$ характерное время жизни до десорбции, $j_{d i f f}^{s}-$ диффузионный поток адатомов в каплю с поверхности подложки, $j_{d i f f}^{f}-$ диффузионный поток в каплю с боковой поверхности ННК (размерности $\mathrm{s}^{-1}$ ). Таким образом, размерность выражения в правой части $(1)-\mathrm{nm} / \mathrm{s}$. Поверхностная концентрация $n_{l}$ возрастает при уменьшении радиуса 


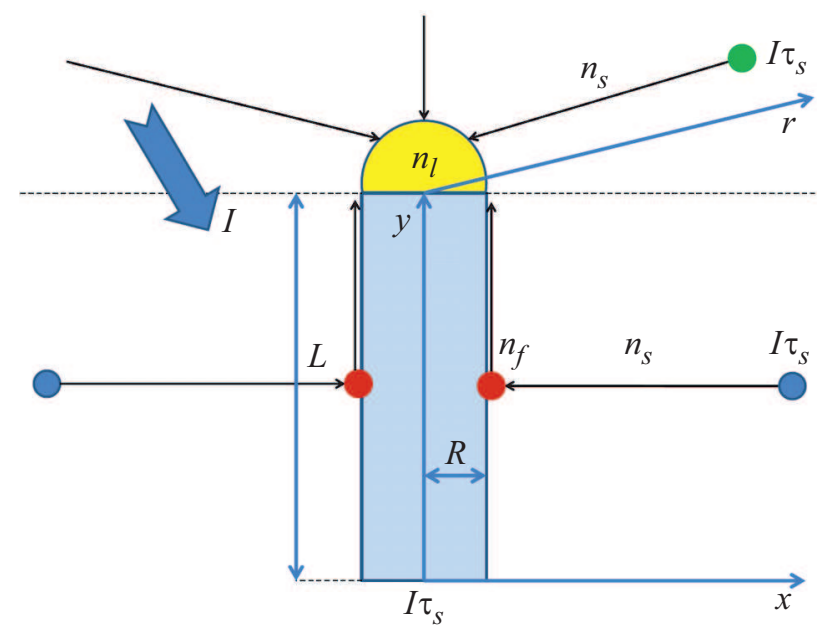

Рис. 1. Иллюстрация роста планарного ННК по механизму ПЖК. ННК растет из капли катализатора вдоль оси $y$. Вклады в скорость роста ННК включают адсорбцию-десорбцию на поверхности капли, диффузию непосредственно в каплю вдоль радиус-вектора $r$, диффузию атомов, попадающих непосредственно на боковую поверхность ННК (с концентрацией $n_{f}$ ), и диффузию адатомов, собранных с поверхности подложки (с концентрацией $n_{s}$ ). Скорость осаждения элемента, лимитирующего рост, равна $I$; концентрация адатомов вдали от капли равна $I \tau_{s}$ и обращается в $n_{l}$ на границе с каплей.

капли за счет эффекта Гиббса-Томсона, связанного с увеличением химического потенциала атомов капли при уменьшении ее радиуса $[14,15,18]$ :

$$
n_{l}=n_{l}^{\infty} \exp \left(\frac{R_{\mathrm{GT}}}{R}\right) .
$$

Здесь $n_{l}^{\infty}$ - равновесная концентрация без учета кривизны поверхности, $R_{\mathrm{GT}}$ - характерный радиус Гиббса-Томсона, пропорциональный поверхностной энергии капли.

Прямой диффузионный поток в каплю вычисляется согласно выражению

$$
j_{d i f f}^{s}=\left.\pi R D_{s} \frac{d n_{s}}{d r}\right|_{r=R}
$$

где $D_{s}$ - коэффициент диффузии адатомов по поверхности подложки, $n_{s}$ - их концентрация. Вблизи капли предполагаем, что градиент концентрации адатомов направлен к центру капли. Тогда их концентрацию можно считать зависящей только от расстояния до центра капли $r$. Для $n_{s}$ в стационарном режиме имеем уравнение, подобное приведенному в $[17,20]$ :

$$
\begin{aligned}
& D_{s}\left(\frac{d^{2} n_{s}}{d r^{2}}+\frac{1}{r} \frac{d n_{s}}{d r}\right)+I-\frac{n_{s}}{\tau_{s}}=0, \\
& n_{s}(r \rightarrow \infty)=I \tau_{s}, \quad n_{s}(r=R)=n_{l}
\end{aligned}
$$

(с граничными условиями, предполагающими равенство концентрации адатомов $I \tau_{s}$ на бесконечности и концентрации в жидкости на границе с каплей). Решение данной задачи дает прямой диффузионный поток

$$
j_{\text {diff }}^{s}=\pi R \lambda_{s} I\left(1-\frac{n_{l}}{I \tau_{s}}\right) \frac{K_{1}\left(R / \lambda_{s}\right)}{K_{0}\left(R / \lambda_{s}\right)},
$$

где $\lambda_{s}=\sqrt{D_{s} \tau_{s}}$ - диффузионная длина адатома на поверхности подложки, $K_{i}$ - модифицированные функции Бесселя порядка $i$.

Для достаточно длинного ННК можно ограничиться одномерной задачей для диффузии адатомов с поверхности подложки на боковую поверхность ННК вдоль оси $x$. Соответствующее уравнение для адатомов вдали от концов ННК имеет вид

$$
\begin{gathered}
D_{s} \frac{\partial n_{s}}{\partial x^{2}}+I-\frac{n_{s}}{\tau_{s}}=0, \\
n_{s}(x \rightarrow \infty)=I \tau_{s}, \quad n_{s}(x=R)=n_{f}(y) .
\end{gathered}
$$

Второе граничное условие предполагает равенство поверхностной концентрации неизвестной пока концентрации на боковой поверхности ННК, которая зависит от координаты $y$ вдоль оси ННК. Отсюда возникает параметрическая зависимость от у для соответствующего диффузионного потока. Диффузионное поступление адатомов дает поток

$$
I_{s}=\left.\frac{2 D_{s}}{\pi R} \frac{d n_{s}}{d x}\right|_{x=R} .
$$

Решая уравнение (6), из (7) получаем

$$
I_{s}=\frac{2 I \lambda_{s}}{\pi R}\left[1-\frac{n_{f}(y)}{I \tau_{s}}\right] .
$$

В случае одномерной диффузии по боковой поверхности ННК имеем уравнение для концентрации адатомов $n_{f}$ вида

$$
\begin{gathered}
D_{f} \frac{d^{2} n_{f}}{d y^{2}}+I+I_{s}-\frac{n_{f}}{\tau_{f}}=0, \\
n_{f}(y=0)=I \tau_{s}, \quad n_{f}(y=L)=n_{l} .
\end{gathered}
$$

Здесь учтен дополнительный диффузионный поток, определяемый выражением (7). На другом конце ННК (без капли) ставим то же граничное условие, что и на бесконечном расстоянии от ННК, а на границе с каплей концентрация адатомов должна переходить в концентрацию атомов в жидкости. Результирующий поток с боковой поверхности на вершину ННК рассчитывается согласно выражению

$$
j_{\text {diff }}^{f}=-\left.\pi R \frac{d n_{f}}{d y}\right|_{y=L} .
$$

Решение данной задачи имеет вид

$$
\begin{aligned}
j_{\text {diff }}^{f}= & \frac{\pi R D_{f}}{\Lambda}\left\{\left(\tilde{I} \tilde{\tau}-I \tau_{s}\right)[1-\operatorname{cth}(L / \Lambda)] e^{-L / \Lambda}\right. \\
& \left.+\left(\tilde{I} \tilde{\tau}-n_{l}\right) \operatorname{cth}(L / \Lambda)\right\},
\end{aligned}
$$

где

$$
\tilde{I}=I\left(1+\frac{2 \lambda_{s}}{\pi R}\right), \quad \frac{1}{\tilde{\tau}}=\frac{1}{\tau_{f}}+\frac{2 \lambda_{s}}{\pi R} \frac{1}{\tau_{s}},
$$


$\Lambda=\sqrt{D_{f} \tilde{\tau}}-$ перенормированная диффузионная длина адатома на боковой поверхности ННК. Предполагая $\tau_{f}=\tau_{s}$, получаем $\tilde{I} \tilde{\tau}=I \tau_{s}$, что соответствует равенству нулю первого слагаемого в выражении (11). При этом $\Lambda=\lambda_{f} / \sqrt{1+2 \lambda_{s} /(\pi R)}$, где $\lambda_{f}=\sqrt{D_{f} \tau_{s}}=\sqrt{D_{f} \tau_{f}}-$ диффузионная длина на боковой поверхности ННК.

Собирая все вклады вместе, из уравнений (1), (2), (5) и (11) (при $\tau_{f}=\tau_{s}$ ) получаем основной результат работы

$$
\begin{aligned}
& \frac{d L}{d t}=2 \Omega I\left\{1-\frac{n_{l}^{\infty}}{I \tau_{l}} e^{R_{\mathrm{GT}} / R}+\left(1-\frac{n_{l}^{\infty}}{I \tau_{s}} e^{R_{\mathrm{GT}} / R}\right)\right. \\
& \left.\times\left[\frac{\lambda_{s}}{R} \frac{K_{1}\left(R / \lambda_{s}\right)}{K_{0}\left(R / \lambda_{s}\right)}+\frac{\lambda_{f}}{R} \sqrt{1+\frac{2 \lambda_{s}}{\pi R}} \operatorname{cth}\left(\frac{L}{\Lambda}\right)\right]\right\} .
\end{aligned}
$$

Анализ полученного уравнения для скорости роста планарных ННК приводит к следующим выводам. Во-первых, скорость роста обращается в нуль при некотором минимальном радиусе за счет эффекта Гиббса-Томсона, как и для вертикальных ННК [18]. Во-вторых, скорость роста достаточно коротких ННК зависит от их длины $L$, общий вид зависимости длины ННК от радиуса и прочих параметров проанализирован в работе [11]. При $L \gg \Lambda$ зависимость от длины исчезает. В-третьих, зависимость длины от радиуса ННК представляет собой функцию с максимумом, возрастающую при малых и убывающую при больших $R$, что характерно для диффузионного механизма роста [16-18]. Характер диффузионной зависимости скорости роста планарных ННК от $R$ отличается, однако, от случая вертикального ПЖК-роста.

При малых диффузионных длинах адатомов на поверхности подложки $\left(\lambda_{s} / R \ll 1\right)$ имеем $K_{1}\left(R / \lambda_{s}\right) / K_{0}\left(R / \lambda_{s}\right) \cong 1$ и $\sqrt{1+2 \lambda_{s} /(\pi R)} \cong 1$. В этом случае диффузионный вклад в скорость роста в уравнении (13) переходит в классическую зависимость $1 / R[4,16,17]$. В противоположном случае $\left(\lambda_{s} / R \gg 1\right)$ имеем

$$
\begin{gathered}
K_{1}\left(R / \lambda_{s}\right) / K_{0}\left(R / \lambda_{s}\right) \cong \lambda_{s} /\left[R \ln \left(\lambda_{s} / R\right)\right], \\
\sqrt{1+2 \lambda_{s} /(\pi R)} \cong \sqrt{2 \lambda_{s} /(\pi R)} .
\end{gathered}
$$

Следовательно, убывающая зависимость от радиуса в уравнении (13) содержит два вклада: $1 / R^{2}$ с малой логарифмической поправкой и $1 / R^{3 / 2}$ (последний наблюдается только для планарных ННК). В результате скорость роста длинных планарных ННК (при $L \gg \Lambda$ ) может быть представлена в виде приближенного выражения

$$
\frac{d L}{d t} \cong 2 \Omega I\left\{1-\theta_{l v} e^{R_{\mathrm{GT}} / R}+\left(1-\theta_{l s} e^{R_{\mathrm{GT}} / R}\right)\left(\frac{\lambda}{R}\right)^{m}\right\} .
$$

Здесь $\theta_{l v}=n_{l}^{\infty} /\left(I \tau_{l}\right), \theta_{l s}=n_{l}^{\infty} /\left(I \tau_{s}\right)$, а показатель $m$ принимает значения $1,3 / 2$ или 2 в зависимости от соотношения между диффузионными длинами и радиусом

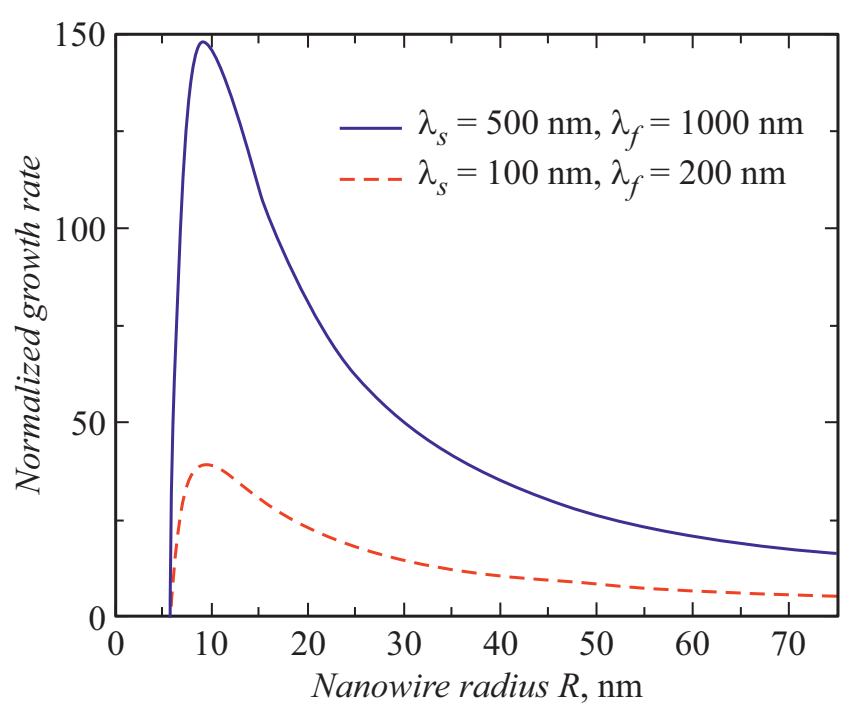

Рис. 2. Зависимости приведенной скорости роста планарных ННК $(1 / 2 \Omega I) d L / d t$ от радиуса, полученные на основе выражения (13) при $L \gg \Lambda, n_{l}^{\infty} /\left(I \tau_{l}\right)=n_{l}^{\infty} /\left(I \tau_{s}\right)=0.5, R_{\mathrm{GT}}=4 \mathrm{~nm} \mathrm{в}$ случае больших ( $\lambda_{s}=500 \mathrm{~nm}, \lambda_{f}=1000 \mathrm{~nm}$, сплошная линия) и малых ( $\lambda_{s}=100 \mathrm{~nm}, \lambda_{f}=200 \mathrm{~nm}$, штриховая линия) диффузионных длин.

ННК. Типичные зависимости скорости роста от радиуса ННК, приведенные на рис. 2 в случае больших и малых диффузионных длин, иллюстрируют описанные выше закономерности.

Таким образом, предложено уравнение, описывающее скорость роста планарных ННК и конкуренцию между эффектами Гиббса-Томсона в капле и поверхностной диффузией, что приводит к немонотонной зависимости длины ННК от радиуса. Механизмы диффузионного роста планарных ННК отличаются от таковых для вертикальных, что приводит к различию в степенных показателях зависимости скорости роста от радиуса. Упрощенное уравнение (14) хорошо описывает данные по кинетике выращивания планарных $\mathrm{ZnSe}$ и $\mathrm{ZnS}$ с $\mathrm{Au-катализаторами} \mathrm{методом} \mathrm{газофазной} \mathrm{эпитаксии} \mathrm{[11].}$ Более точное уравнение (13) может быть использовано для моделирования и управления планарным ростом ННК в различных системах материалов и на различных поверхностях.

\section{Финансирование работы}

Работа выполнена при поддержке Российского фонда фундаментальных исследований (гранты № 20-52-16301, 20-02-00351, 19-52-53031 и 18-02-40006).

\section{Конфликт интересов}

Авторы заявляют, что у них нет конфликта интересов. 


\section{Список литературы}

[1] Zhang A., Zheng G., Lieber C.M. Nanowires: building blocks for nanoscience and nanotechnology. Springer, 2016. $327 \mathrm{p}$.

[2] Wagner R.S., Ellis W.C. // Appl. Phys. Lett. 1964. V. 4. P. 89-90.

[3] Colombo C., Spirkoska D., Frimmer M., Abstreiter G., Fontcuberta $i$ Morral A. // Phys. Rev. B. 2008. V. 77. P. 155326.

[4] Yang P., Yan P.R., Fardy M. // Nano Lett. 2010. V. 10. P. $1529-1536$.

[5] Yu L., Alet P.-J., Picardi G., Roca i Cabarrocas P. // Phys. Rev. Lett. 2009. V. 102. P. 125501.

[6] Fortuna S.A., Wen J., Chun I.S., Li X. // Nano Lett. 2008. V. 8. P. $4421-4427$.

[7] Friedl M., Cerveny K., Weigele P., Tütüncüoglu G., MartiSanchez S., Huang C., Patlatiuk T., Potts H., Sun Z., Hill M.O., Güniat L., Kim W., Zamani M., Dubrovskii V.G., Arbiol J., Lauhon L.J., Zumbühl D.M., Fontcuberta $i$ Morral A. // Nano Lett. 2018. V. 18. P. 2666-2671.

[8] Tsivion D., Joselevich E. // J. Phys. Chem. C. 2014. V. 118. P. 19158.

[9] Oksenberg E., Popovitz-Biro R., Rechav K., Joselevich E. // Adv. Mater. 2015. V. 27. P. 3999-4005.

[10] Reut G., Oksenberg E., Popovitz-Biro R., Rechav K., Joselevich E. // J. Phys. Chem. C. 2016. V. 120. P. 17087.

[11] Rothman A., Dubrovskii V.G., Joselevich E. // PNAS. 2020. V. 117. P. $152-160$.

[12] Nikoobakht B., Michaels C.A., Stranick S.J., Vaudin M.D. // Appl. Phys. Lett. 2004. V. 85. P. 3244-3246.

[13] Shen Y., Chen R., Yu X., Wang Q., Jungjohann K.L., Dayeh S.A., $W u$ T. // Nano Lett. 2006. V. 16. P. 4158-4165.

[14] Zi Y., Jung K., Zakharov D., Yang C. // Nano Lett. 2013. V. 13. P. 2786-2791.

[15] Дубровский В.Г., Сибирев Н.В., Цырлин Г.Э. // Письма в ЖТФ. 2004. Т. 30. В. 16. C. 41-50.

[16] Seifert W., Borgstrom M., Deppert K., Dick K.A., Johansson J., Larsson M.W., Mårtensson T., Sköld N., Svensson C.P.T., Wacaser B.A., Wallenberg L.R., Samuelson L. // J. Cryst. Growth. 2004. V. 272. P. 211-220.

[17] Dubrovskii V.G., Soshnikov I.P., Sibirev N.V., Cirlin G.E., Ustinov V.M. // J. Cryst. Growth. 2006. V. 289. P. 31-36.

[18] Fröberg L.E., Seifert W., Johansson J. // Phys. Rev. B. 2007. V. 76. P. 153401.

[19] Dubrovskii V.G., Grecenkov J. // Cryst. Growth Design. 2015. V. 15. P. 340-347.

[20] Dubrovskii V.G. // Phys. Status Solidi B. 1992. V. 171. P. 345-356. 